\title{
NOTICIAS SOBRE LOS CATÁLOGOS MONUMENTALES DE ESPAÑA EN EL EPISTOLARIO DE JOSÉ GESTOSO $(1852-1917)$
}

\author{
Carmen de Tena Ramírez \\ Universidad de Sevilla \\ cdetena@us.es
}

\section{RESUMEN}

Este trabajo tiene como objetivo dar a conocer noticias inéditas sobre la elaboración de los catálogos monumentales de España. Estas han sido localizadas en el epistolario del erudito sevillano José Gestoso, conservado en la Institución Colombina de Sevilla, y se han extraído de las cartas que recibía este personaje de parte de algunos de sus autores. Los datos que se dan a conocer ayudan a trazar la intrahistoria de uno de los proyectos más relevantes emprendidos en España, en favor del conocimiento y de la protección del patrimonio histórico-artístico. Al tratarse de documentación de índole privada, se percibe el lado más humano de las tareas de catalogación, y cómo las concibieron y las interpretaron personal e individualmente sus protagonistas.

Palabras Clave: historia del arte en España, fuentes para la historia del arte, historiografía artística, Manuel Gómez-Moreno Martínez, conservación del patrimonio histórico.

\author{
NEWS ABOUT THE MONUMENTAL CATALOGUES \\ OF SPAIN IN THE EPISTOLARY OF JOSÉ GESTOSO \\ (1852-1917)
}

\section{Abstract}

The aim of this paper is to reveal unpublished news on the production of Spain's monumental catalogues. This information has been located in the epistolary collection of the Sevillian scholar José Gestoso, kept at the Institución Colombina in Seville. This information has been extracted from the letters this personality received from some of his authors. It helps to trace the intrahistory of one of the most important projects undertaken in Spain in favour of the knowledge and protection of historical and artistic heritage. Given the private nature of the documentation, the more human side of the cataloguing tasks is perceived, and how they were conceived and interpreted personally and individually by their protagonists.

Keywords: History of Art History in Spain, sources for Art History, Art Historiography, Manuel Gómez-Moreno Martínez, conservation of cultural heritage. 
El fondo documental del erudito sevillano José Gestoso y Pérez (18521917), conservado en la Institución Colombina, es una rica fuente de información para el estudio de la historia y del arte español ${ }^{1}$. Una de sus secciones más valiosas es el epistolario de este personaje, en el que se recogen más de seis mil cartas dirigidas, en su inmensa mayoría, al propio Gestoso. Hace unos años se publicó un artículo en el que se daba a conocer este valioso acervo documental (De Tena Ramírez 2018), y donde además se subrayaba la relevancia que tiene para los investigadores de la historiografía, especialmente artística o arqueológica. Se debe tener en cuenta que Gestoso conoció e incluso entabló amistad con los estudiosos de la historia, la arqueología y el arte español más reconocidos de la época. Hablamos de personajes de la talla de José Ramón Mélida, Manuel Gómez-Moreno Martínez, Vicente Lampérez, Rodrigo Amador de los Ríos, Pierre Paris, August L. Mayer, Arthur Engel, Jorge Bonsor o Elías Tormo, entre otros.

Este epistolario da comienzo en el año 1875 y abarca hasta la muerte de su poseedor en 1917; estos años, que se integran dentro del periodo de la Restauración borbónica, engloban una época de grandes cambios en el panorama cultural español, entre los que destaca especialmente la profesionalización de los estudios histórico-artísticos y arqueológicos. La institucionalización de la historia, la historia del arte y la arqueología se desarrolló en paralelo a las labores de estudio sistemático del patrimonio cultural español, en buena parte propiciadas por las pérdidas que habían marcado el curso del siglo xIx. Tampoco debe menospreciarse como factor de desarrollo la expansión del turismo cultural. Sobre estas y otras muchas cuestiones se debate en las cartas enviadas a Gestoso, y es por esta variedad de temas y enfoques, y por el prestigio de los interlocutores, por lo que su epistolario es una fuente de primer nivel para los estudios histórico-artísticos y arqueológicos en España.

En este estudio queremos centrarnos en uno de los proyectos culturales de mayor relevancia que se promovieron en nuestro país a principios del siglo xx: la formación del catálogo monumental y artístico ${ }^{2}$. Es bien conocida esta empresa, que dio comienzo con la firma del real decreto correspondiente de 1 de junio de 1900, y que, como sabemos, fue una propuesta del ministro de Instrucción Pública y Bellas Artes, Antonio García Alix, a instancias del entonces director de la Real Academia de San Fernando, Juan Facundo Riaño (López-Ocón 2012). A propuesta de este último, el proyecto contó en sus primeros años con Manuel Gómez-Moreno Martínez como el único encargado de llevar a cabo este estudio sistemático y ordenado de la riqueza patrimonial de España. Sin embargo, dada la carga de trabajo que esta empresa suponía y la imposibilidad de que la pudiera llevar a término una sola persona en un tiempo prudencial, desde 1902 se fueron incorporando progresivamente al proyecto otras personas. Esta división del trabajo conllevó «desigualdad de criterios en la recopilación de datos, producto de la dispar cualificación e interés por

${ }^{1}$ Sobre este personaje, véase Casquete de Prado (2016).

2 El proyecto del catálogo monumental de España ha sido tratado por diversos autores, y, por la visión general que aportan, destacamos las publicaciones de López-Yarto (2010, 2012). 
el tema de las personas a quienes se encomendó» (Morales 1996). Efectivamente, y dado que aún no se habían profesionalizado los estudios histórico-artísticos, aún no existían personas plenamente cualificadas, exceptuando a Gómez-Moreno, para, como seńala el citado real decreto, poder «llevar á efecto labor de método que permita llegar á poseer un Catálogo completo de todo aquello que revista en la Historia ó en el Arte un reconocido mérito». Y aunque el plan de trabajo expuesto en el primer catálogo que presentó el investigador granadino (Ávila) fue valorado y reconocido, como veremos con posterioridad, no influyó lo suficiente como para que con él se estableciera un método concreto para catalogar todas las provincias; tampoco el ministerio se preocupó por establecer las normas y las reglas necesarias que esta tarea demandaba (Muñoz Cosme 2012).

Los participantes en el proyecto de los catálogos monumentales eran propuestos por la Comisión Mixta de las Comisiones Provinciales de Monumentos, que agrupaba miembros de la Real Academia de Bellas Artes de San Fernando y de la Historia. Es por esta razón po lo que los nombramientos surgían entre los individuos de estas academias, ya fueran numerarios o correspondientes. También, y según el artículo sexto del real decreto de 14 de febrero de 1902, que modificaba al anterior de 1900, podrían requerirse los servicios de los miembros del cuerpo de Archiveros, Bibliotecarios y Anticuarios. No obstante, la pertenencia a este o a las citadas instituciones no siempre iba aparejada a una formación adecuada en materia histórico-artística, y raramente poseían nociones sobre conservación y restauración de monumentos; así lo ha señalado López-Yarto (2012), que, como buena conocedora de los catálogos monumentales y de sus autores, ha subrayado que los retrasos en la conclusión del proyecto llevaron a incurrir en la «relajación de la exigencia de conocimiento en historia del arte a la hora de proponer a los catalogadores». Como consecuencia de esta falta de formación en la materia, cada autor tendía a centrarse en los temas en los que era experto, no atendiendo a otros aspectos a los que era ajeno (Hidalgo 2012).

La nómina de catalogadores es extensa, tanto como los diferentes grados de calidad que sus trabajos alcanzaron, pero lo que nos compete en esta ocasión es que algunos de ellos conocieron a José Gestoso, siendo incluso amigos suyos. A través de su correspondencia se pueden rastrear noticias y testimonios que estos autores le transmitían acerca de la ejecución del catálogo. La nómina no es amplia, pero la información que ofrecen es muy estimable; estos personajes son Manuel GómezMoreno Martínez (Ávila 1900, Salamanca 1901, Zamora 1903 y León 1906), Rodrigo Amador de los Ríos (Málaga 1907, Huelva 1908 y Barcelona 1913), José Ramón Mélida (Badajoz 1907 y Cáceres 1914), Rafael Ramírez de Arellano (Córdoba 1902) Enrique Romero de Torres (Cádiz 1907 y Jaén 1913) y Adolfo Fernández Casanova (Sevilla, 1907) ${ }^{3}$. Otros integrantes del proyecto como Francisco Rodrí-

3 Se han indicado los catálogos que elaboraron cada uno de estos investigadores y la fecha de su nombramiento. Para ampliar la información sobre cómo se gestó cada uno, véase López-Yarto (2010, 2012). 
guez Marín (Madrid 1907 y Segovia 1908) o Luis Tramoyeres (Castellón 1912) le dirigieron cartas a Gestoso, que se encuentran en su epistolario, especialmente el primero, que era un buen amigo suyo, pero no mencionan datos sobre el catálogo en ninguna de las cartas conservadas.

Paradójicamente, y a pesar de que no dejaba de recibir noticias sobre el tema, José Gestoso no fue autor de ninguno de los catálogos. Este asunto merece nuestra atención y será tratado en la última parte de este estudio.

No es la primera vez que se acude a fuentes epistolares para completar el conocimiento que se tiene sobre la elaboración de los catálogos monumentales, pues las fuentes oficiales no revelan la intrahistoria de esta iniciativa. López-Yarto, que consultó la correspondencia de Manuel Gómez-Moreno y de su círculo de familiares y amigos, ya advirtió que los datos que nos ofrece la correspondencia son «más expresivos [...] que los fríos documentos de los archivos» (López-Yarto 2010). Por otro lado, en los últimos años se ha publicado un libro con el epistolario de Manuel Gómez-Moreno y su mujer Elena Rodríguez Bolívar, durante la elaboración del catálogo de Zamora, complementado con varios estudios sobre esta etapa de la vida del historiador (Lorenzo y Pérez 2017).

Nuestro repaso a las noticias encontradas sobre los catálogos monumentales en el epistolario de José Gestoso comienza con Manuel Gómez-Moreno Martínez. Este investigador granadino ha sido considerado uno de los padres de la disciplina de la historia del arte en España junto con Elías Tormo (Borrás Gualís 2006). Es el autor de los catálogos que más información reveló a Gestoso sobre los mismos, y gracias a la conservación de su epistolario tenemos muchas noticias sobre cómo transcurrió la investigación de los que le fueron encomendados. Alfredo Mederos ha investigado cómo se gestó el nombramiento de Gómez-Moreno como encargado del proyecto del catálogo monumental (Mederos Martín 2015) y de las suspicacias que este levantó, especialmente debido a una posible injerencia por parte de Juan Facundo Riaño. Sin duda, la amistad previa entre estos personajes apuntaba a un posible favoritismo, pero la sobresaliente labor del joven investigador granadino acabaría demostrando su valía, y que su elección estaba plenamente justificada (Mederos Martín 2018).

De sus primeros meses de trabajo en Ávila le dijo a Gestoso: «Aquí me tiene enfrascado en esta labor tan ingrata por lo aperreada y tan larga y penosa si ha de llevarse á punta de lanza como deseo: es cuestión de honra y de amor propio, no de medro, y así, antes de echarme atrás aquí me dejo las asaduras» ${ }^{4}$. Sus palabras demuestran que habían sido muchas las confianzas depositadas en él por su valedor, Riaño, y que no podía desfallecer a pesar de la dureza de la tarea. Ciertamente, las complicaciones aparejadas al proyecto y la riqueza del tesoro artístico nacional no resultaban halagüeñas a la hora de pretender cumplir el objetivo de catalogar el

${ }^{4}$ Biblioteca Capitular y Colombina, Fondo Gestoso (en adelante BCC, FG), Correspondencia, 1899-1900, n. ${ }^{\circ}$ 224, carta de Manuel Gómez-Moreno a José Gestoso, fechada en Arévalo el 8/10/1900. El contenido de todas las cartas se ha transcrito respetando la grafía original. 
patrimonio de todas las provincias espańolas. Por esta razón, como ya se ha indicado, hubo que modificar el real decreto de 1 de junio de 1900 con el de 14 de febrero de 1902, para así abrir el proyecto a más autores. Los siguientes nombramientos no tardarían en llegar, y Gómez-Moreno quiso poner al corriente de ello a Gestoso:

Ya sabrá V. que por empeños especiales se ha encomendado el Catálogo de Guadalajara al Sr. Catalina y el de Córdoba á Ramírez de Arellano; quizá V. conozca mejor que yo á ambos y pueda juzgar si es ó no acertada la elección; yo realmente no lo se y espero muy deseoso el parto de sus facultades. A Arellano he tenido ocasión de conocerlo ahora ligeramente; me parece que es como me lo figuraba, y no como yo lo desearía, ciertamente; Catalina es mi superior jerárquico, y basta. Pero así en confianza, le diré, que dentro del terreno puramente arqueológico y artístico no les temo, ó más bien temo que no me satisfagan; pues crea V. que tengo verdadera ansia de ver á otro salir al camino y que me acompañe al paso, ó que se me adelante tanto mejor ${ }^{5}$.

Se refería a Juan Catalina García, que al igual que Riaño era profesor de la Escuela Superior de Diplomática de Madrid, centro de formación de los archiveros, bibliotecarios y anticuarios. Sin embargo, y a pesar del cargo y de otras distinciones, no tenía el nivel de Gómez-Moreno, y este lo sabía. Tampoco era especialmente brillante Rafael Ramírez de Arellano aunque parece que Gestoso lo valoraba, pues en una carta, siguiente a la anterior, le decía Gómez-Moreno: «Me alegro de saber por V. su capacidad $»^{6}$.

El sentimiento era mutuo, pues Ramírez de Arellano no tenía en gran consideración a Gómez-Moreno, o mejor dicho, a su trabajo. Ello pudo estar influenciado por el hecho de que a este le pagaban 800 pesetas al mes, mientras que a Ramírez de Arellano solo 500, al parecer, porque no había crédito para más. Si bien valoraba el catálogo de Ávila, esto era por su método, que era el que creía que debían seguir todos los autores; un método del que opina "no es suyo, sino que se lo dio Riaño». La crítica más dura hacia este catálogo se refiere a lo que para Ramírez de Arellano es un exceso de documentación gráfica: «Puedo decir que es una lista de objetos con numerosísimas fotografías. Los que lo califican de notable no han visto más que las estampas y los que han examinado el texto creen que vale muy poco [...]. En su trabajo no hay casi nada de historia y nada de descripción »?. A este respecto habría que añadir que el catálogo de Córdoba lo entregó este autor sin fotografías, no porque no las creyera necesarias, sino porque había cobrado 300 pesetas mensuales menos, por lo que consideró más adecuado pasar este encargo al editor del catálogo ${ }^{8}$.

BCC, FG, Correspondencia, 1901-1902, n. ${ }^{\circ} 200$, carta de Manuel Gómez-Moreno a José Gestoso, fechada el 10/5/1902.

${ }^{6}$ BCC, FG, Correspondencia, 1901-1902, n. ${ }^{\circ}$ 273, carta de Manuel Gómez-Moreno a José Gestoso, fechada el 10/11/1902.

${ }^{7}$ BCC, FG, Correspondencia, 1903-1904, n. ${ }^{\circ}$ 95, carta de Rafael Ramírez de Arellano a José Gestoso, fechada en Córdoba el 8/5/1903.

${ }^{8}$ Algo que no sucedió hasta 1983, en edición anotada por José Valverde Madrid. 
Muy al contrario del criterio expresado por Ramírez de Arellano, el catálogo de Ávila de Gómez-Moreno es de los más valorados de toda la colección, especialmente por la presencia de fotografías, que desde el principio, fueron para él indispensables, por lo que tuvo que adquirir una cámara de fotos para llevarla en sus excursiones:

La catalogación había de hacerse directamente recorriendo y fotografiando toda la provincia. Había de proveerse de máquina fotográfica, grave asunto, pues era cara y su pequeño sueldo de profesor de Arqueología en el Sacromonte granadino no daba mucho de sí. Al fin encontró una, cara (500 pesetas), pero apropiada (Gómez-Moreno 1983).

Esta inversión supuso más de la mitad de su asignación mensual como autor del catálogo. Eso sí, antes de realizarla estuvo practicando con una cámara de Juan Facundo Riaño hasta que dominó el procedimiento (Gómez-Moreno 1995).

No es baladí el hecho de que tras la entrega del catálogo de Ávila en 1901, se estableciera en el ya citado real decreto de 1902 que «la descripción de los objetos o monumentos se presentará ilustrada con planos, dibujos o fotografías». Con esta disposición queda claro que se reconocía por primera vez y de manera oficial la importancia que tenía la fotografía como medio de estudio de la historia del arte y de conocimiento del patrimonio (López-Yarto 2010). Pero, como ha señalado Argerich (2012), en este reconocimiento hubo de ser decisivo el modelo propuesto por Gómez-Moreno para Ávila, que ya había sido entregado y, desde luego, apreciado en el ministerio.

La mayor parte de los autores presentaron los catálogos con fotografías, aunque solo las realizaron de manera sistemática y personalmente Manuel GómezMoreno y Juan Cabré. Este primer investigador tomó casi todas sus imágenes, pero por ejemplo en Ávila recibió la ayuda del profesional Casiano Alguacil (fig. 1), un seguidor de la producción de Jean Laurent, y que, como él, llevaba años dedicado a la fotografía artística (Argerich 2012). Al margen de esta excepción, parece que él fue el único autor de las instantáneas, tal y como indica en el catálogo de León o como le hizo saber a Gestoso?.

Otros autores también revelaron al erudito sevillano su interés por la inclusión de fotografías, como fue el caso de su buen amigo Adolfo Fernández Casanova. Este arquitecto se había ocupado de la restauración de la catedral de Sevilla y de la Giralda, y en la Junta de Obras de esta coincidieron ambos personajes, que desde entonces se profesaron gran afecto. Esta cercanía a Sevilla y el conocimiento de su patrimonio arquitectónico le habría motivado a solicitar en 1907 la labor de catalogación de esta provincia, en la que, veremos, contó con la ayuda de Gestoso. De vuelta al tema que referíamos en líneas anteriores, la fotografía, decía Fernández Casanova que durante sus excursiones habían tenido «mala estrella mi sobrina y

9 De León dice haber sacado 350 fotografías, él mismo, BCC, FG, Correspondencia, 1909 vol. 2, n. ${ }^{\circ}$ 109, carta de Manuel Gómez-Moreno a José Gestoso, fechada en Granada el 10/10/1909. 


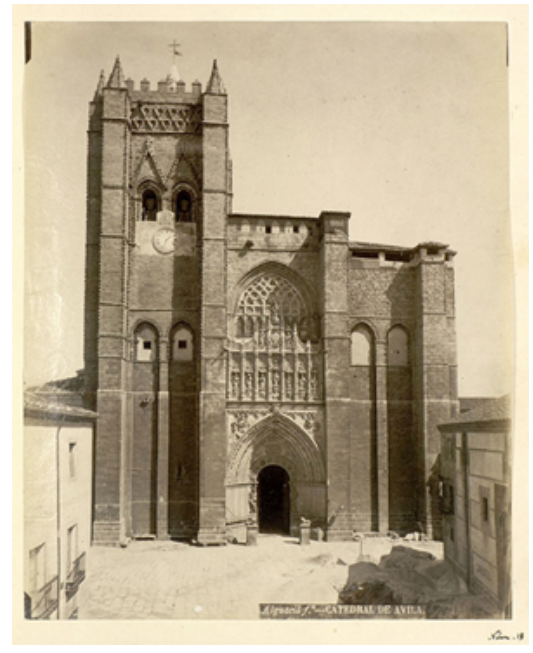

Fig. 1. Casiano Alguacil (fot.), Catedral de Ávila, ca. 1900. Catálogo monumental de España.

Provincia de Ávila, tomo 1 de fotografías, n. ${ }^{\circ}$ 18. (C)SIC, CCHS,

Biblioteca Tomás Navarro Tomás.

yo, pues se nos descompuso por 3 veces la máquina por no haberla arreglado bien». Por esta razón, decía de las fotografías que «algunas las sacamos nosotros, otras las he mandado hacer a los fotógrafos; unas adquiriendo los clichés y otras comprando dos positivos de cada vista, para quedar yo con copia de todo ${ }^{10}$.

Solo los catálogos de Córdoba, Guadalajara y Huesca no se presentaron con fotografías (Argerich 2012). Ramírez de Arellano adujo que el Estado pagaba mal, por eso no incluyó fotografías; eso sí, reconoció que suponía para él tal honor que podría haber hecho el catálogo de Córdoba gratis ${ }^{11}$. Otros catalogadores tuvieron que invertir su propio dinero para lograr un resultado satisfactorio, como le ocurrió a Enrique Romero de Torres. Este cordobés reconoció haber tenido que hacer frente a varios gastos asociados a su elaboración: «Me ha costado el dinero como tendrá que costarle a todo el que quiera hacer esta clase de trabajos a conciencia y recorra pueblo por pueblo, aldea por aldea, toda una provincia como yo, pero no me pesa, por haber salido airoso de mi empresa» ${ }^{12}$. Reconocía, pues, la necesidad de visitar todos los rincones de la provincia, de la misma forma que hizo Manuel Gómez-Moreno

${ }^{10}$ BCC, FG, Correspondencia, cartas de Adolfo Fernández Casanova a José Gestoso, n. ${ }^{\circ}$ 170, carta de Adolfo Fernández Casanova a José Gestoso, fechada en Madrid el 28 de abril de 1908.

${ }^{11}$ BCC, FG, Correspondencia, 1903-1904, n. ${ }^{\circ}$ 95, carta de Rafael Ramírez de Arellano a José Gestoso, fechada en Córdoba el 08/5/1903.

${ }_{12}$ BCC, FG, Correspondencia, 1909 vol. 2, n. ${ }^{\circ} 109$, carta de Enrique Romero de Torres a José Gestoso, fechada en Córdoba el 17/9/1909. 
o como le recomendaba José Ramón Mélida a Adolfo Fernández Casanova, si bien este último no estaba del todo convencido de que esa opción fuera plausible, dada la riqueza monumental de la provincia de Sevilla:

El amigo Mélida me decía anoche que debía yo verlo todo por mí mismo; pero no comprende que tengo yo una provincia excepcional. A pesar de los muchos datos que usted sabe que yo ya tenía, he andado ahora yo corriendo antes un mes y ahora otro por los pueblos, sin contar los viajes a Carmona, y le aseguro a V. por lo que he visto del catálogo de Ávila que sin duda mucho mejor escrito que será el mío, porque su autor sabe mucho más; pero en cuanto a la extensión del trabajo mío tiene que ocupar muchísimo más volumen ${ }^{13}$.

Conviene subrayar de nuevo que el primer catálogo de Gómez-Moreno era el referente absoluto de los autores, quienes, como puede apreciarse, lo mencionan infravalorándolo, ya fuera por el exceso de fotografías o por la escasa extensión de texto. Al menos fue modelo en cuanto al afán por explorar todo el territorio de la provincia, aunque no todos llegaran a equipararle, como le pasó a Fernández Casanova. A pesar de las limitaciones que la extensión y riqueza de la provincia de Sevilla le suponían, parece que pasó un mes recorriendo Écija, Osuna, Marchena, Morón, Sanlúcar, Cazalla, Alanís y Constantina ${ }^{14}$, esto es, sus pueblos más notables, y siempre acompañado de su sobrina, Sofía Dalda, que, igual que él, había enviudado ${ }^{15}$. En sus cartas a Gestoso se percibe la añoranza que sentía por su mujer, Saturnina de Torre Saldaña, cuya muerte se había producido el 22 de febrero de 1908, poco después de iniciar las tareas de estudio del patrimonio de la provincia. De modo que tuvo que enfrentarse a la tarea de elaboración del catálogo sufriendo el duelo y sin descuidar ni sus obligaciones académicas ni las docentes en la Escuela de Arquitectura de Madrid, aunque le refirió varias veces a su amigo que los quehaceres diarios le ayudaban a mitigar el dolor por tan terrible pérdida.

La compañía de familiares era un sólido apoyo emocional para los autores del catálogo, como lo fue Sofía Dalda para el recién viudo Fernández Casanova. Por eso era habitual que viajaran acompañados; pocos de ellos vivían en la provincia que se les había asignado, y por esta razón tenían que trasladarse desde su domicilio habitual. Manuel Gómez-Moreno estuvo acompañado en algunos de sus viajes por su mujer, Elena Rodríguez Bolívar, desde que se casaron en 1903. Ella fue, además de grata compañía, su ayudante en la elaboración de los catálogos de Zamora y León: «Gómez-Moreno cuenta con Elena como amanuense, quien le copia los textos con

${ }_{13}$ El subrayado aparece en el original, BCC, FG, Cartas dirigidas al Excmo. Sr. Gestoso por D. Adolfo Fernández Casanova desde el año 1882 al 1915, n. ${ }^{\circ}$ 170, carta de Adolfo Fernández Casanova a José Gestoso, fechada en Madrid el 28/4/1908.

${ }_{14}$ BCC, FG, Cartas dirigidas al Excmo. Sr. Gestoso..., n. ${ }^{\circ}$ 170, carta de Adolfo Fernández Casanova a José Gestoso, fechada en Madrid el 28/4/1908.

${ }_{15}$ Son muchas las cartas en las que menciona a su sobrina junto a él, por ejemplo al hacer «la peregrinación por los pueblos», cf. BCC, FG, Cartas dirigidas al Excmo. Sr. Gestoso..., n. ${ }^{\circ} 169$, carta de Adolfo Fernández Casanova a José Gestoso, fechada en Morón el 28/3/1908. 
una letra clara y hermosa, inventada para este menester, con abandono de la suya cursiva habitual, y, además, ha aprendido a colaborar en la tarea de hacer pruebas fotográficas» (Gómez-Moreno 1995). El matrimonio incluso entabló amistad con los habitantes de la provincia de Zamora, de donde los despidieron con gran afecto (Lorenzo y Pérez 2017).

El arqueólogo Rodrigo Amador de los Ríos se inició en la tarea catalogadora con la provincia de Málaga, en la que estuvo con su mujer, dos hijas y su hijo Alfonso, que allí los esperaba, destinado en la capital como jefe del Archivo de Hacienda. La razón principal por la que el arqueólogo solicitó este destino fue precisamente de índole familiar; el médico de su hija Elisa le había recomendado viajar a esta tierra para que la niña se repusiera del tifus, enfermedad de la que casi muere $^{16}$. En Huelva, segundo catálogo del que se encargó este personaje, también estuvo acompañado; la presencia de su mujer era prácticamente imprescindible porque se había quedado sordo y necesitaba su auxilio para poder comunicarse: «Como mi sordera malhadada e incorregible me ha convertido en $1 / 4$ de hombre para estos menesteres, me acompañarán, en esa expedición, como en la de Málaga, mi mujer y mi hijo Alfonso» ${ }^{17}$.

La compañía de los seres queridos siempre fue un alivio para los autores, ya que las condiciones de trabajo resultaban casi siempre muy duras de sobrellevar. Del frío experimentado en las excursiones por Ávila contaba Gómez-Moreno a Gestoso: «Yo sigo adelante en mi penosa tarea, ya aquí [en la ciudad de Ávila], ya correteando por los pueblos, y mañana salgo para la última excursión grande que me queda [...]. Allá por Arenas y Cebreros á donde ahora voy, dicen que no hace frío; Dios me la depare buena ${ }^{18}$. El investigador granadino siempre demostraba en sus cartas entereza y buen humor. Tras muchas quejas acerca del trasiego y las condiciones climáticas que conllevaba la ejecución del proyecto, reconocía que este esfuerzo tenía su encanto: «Hermosas obras de arte y una hartada de paisaje compensan el trabajo físico»" ${ }^{19}$.

Por tanto, no le faltaban razones para ironizar con la adjudicación de la provincia de Málaga a Amador de los Ríos: «Un catálogo tiene lo suyo y ya veremos cómo se las arregla el compadre por aquellas serranías de Málaga. Sospecho que se contentará con verlas desde el tren» ${ }^{20}$. Pero no iba desencaminado, pues ya fuera por las dificultades físicas, o bien por la falta de colaboración de la población

${ }^{16}$ BCC, FG, Correspondencia, 1907-1908, n. ${ }^{\circ}$ 17, carta de Rodrigo Amador de los Ríos a José Gestoso, fechada en Málaga el 25/3/1907.

${ }_{17}$ BCC, FG, Correspondencia, 1907-1908, n. ${ }^{\circ} 295$, carta de Rodrigo Amador de los Ríos a José Gestoso, fechada en Madrid el 30/11/1908.

${ }^{18}$ BCC, FG, Correspondencia 1899-1900 , n. ${ }^{\circ}$ 231, carta de Manuel Gómez-Moreno a José Gestoso, fechada en Ávila el 12/11/1900.

${ }_{19}$ BCC, FG,Correspondencia, 1901-1902, n. ${ }^{\circ}$ 181, carta de Manuel Gómez-Moreno a José Gestoso, fechada en Granada el 7/1/1902.

${ }^{20}$ Gestoso era el padrino de bautismo de una de las hijas de Amador de los Ríos, de ahí la denominación de compadre, BCC, FG, Correspondencia, 1907-1908, n. ${ }^{\circ}$ 24, carta de Manuel Gómez-Moreno a José Gestoso, fechada en Granada el 13/4/1907. 
local, muchos autores se habían visto tentados a abandonar el proyecto, y solo su afición por los estudios histórico-artísticos los disuadía. Así lo manifestó Fernández Casanova ante la frustración de no contar con las fotografías que había encargado al fotógrafo Medina: «Si no fuera por la chifladura que tengo por los monumentos de la provincia de Sevilla me arrepentiría de haberme metido en esta barahúnda y pelear con tantos, pasar malos ratos, etc. ${ }^{21}$. Incluso Ramírez de Arellano, que consideraba esta tarea un honor, reconocía que «el trabajo es pesado porque aún conociendo yo todo lo de Córdoba he tenido que volverlo a ver ${ }^{22}$.

No debemos olvidar que los catalogadores no siempre contaban con el apoyo de las autoridades locales, civiles o eclesiásticas. Según dijo Amador de los Ríos a Gestoso, el obispo de Málaga no le autorizó a entrar en los conventos de monjas que hay en la provincia ${ }^{23}$. Más suerte tuvo en cambio en Huelva, pues se publicó en el boletín oficial de esta provincia una circular escrita por el gobernador civil dirigida a los alcaldes de la misma, para que ayudaran al investigador en su tarea (LópezYarto 2010). Este caso fue excepcional, pues la mayoría topó en alguna ocasión con la indiferencia y/o desconfianza de los encargados de tutelar el patrimonio histórico-artístico. Gómez-Moreno tuvo algunos problemas en León: «Tengo allí algunos puntos negros que me inquietan y recelo no poder aclararlos, por encontrarme escollos de personas difíciles de sortear». Al parecer el obispo de allí, Juan Manuel Sanz y Saravia, y su secretario le apoyaron en su tarea, pero no tanto como él necesitaba para poder llevar a cabo una adecuada revisión de las riquezas patrimoniales de la provincia ${ }^{24}$.

José Ramón Mélida fue uno de los afortunados que contaron con la ayuda de la población local, durante su estancia en Badajoz. Este personaje, esencial para el desarrollo de la arqueología científica en España, mantuvo una relación con José Gestoso bastante larga, fundamentada, sobre todo, en las labores de protección del patrimonio histórico, pues Mélida era miembro de la Real Academia de la Historia, y el papel de esta institución en la protección del patrimonio monumental español fue clave.

El primer catálogo monumental que afrontó Mélida fue el de Badajoz, aunque le habían encomendado poco antes el de Tarragona, que no pudo llevar a la práctica. Hizo saber a Gestoso su nombramiento en mayo de 1907 y desde entonces, parece que le estuvo transmitiendo algunos descubrimientos que le pudieran

${ }^{21}$ BCC, FG, Cartas dirigidas al Excmo. Sr. Gestoso..., n. ${ }^{\circ}$ 180, carta de Adolfo Fernández Casanova a José Gestoso, fechada en Madrid el 1/11/1908.

22 BCC, FG, Correspondencia, 1903-1904, n. ${ }^{\circ} 95$ carta de Rafael Ramírez de Arellano a José Gestoso, fechada en Córdoba el 8/5/1903.

23 BCC, FG, Correspondencia, 1907-1908, n. ${ }^{\circ} 23$, carta de Rodrigo Amador de los Ríos a José Gestoso, fechada en Málaga el 12/4/1907.

${ }_{24}$ BCC, FG, Correspondencia, 1907-1908, n. ${ }^{\circ}$ 18, carta de Manuel Gómez-Moreno a José Gestoso, fechada en Granada el 31/3/1907. 


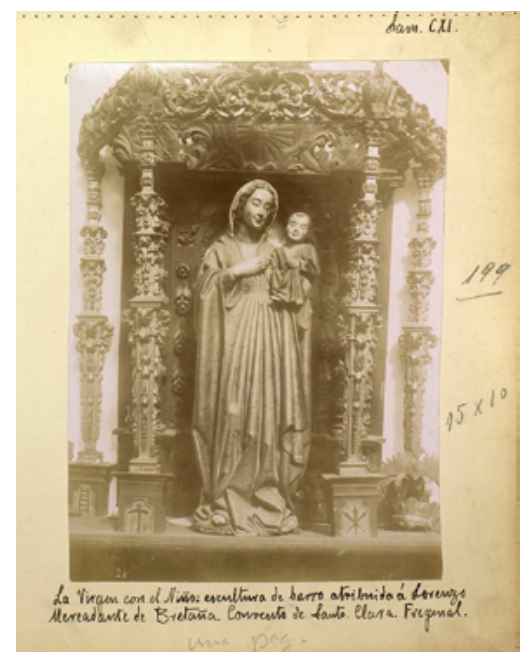

Fig. 2. Fotógrafo desconocido, Virgen con el Niño, ca. 1909. Catálogo Monumental y Artístico de la Provincia de Badajoz, tomo Iv, fotografías, lám. CXI. (CCSIC, CCHS, Biblioteca Tomás Navarro Tomás.

resultar de interés a su amigo sevillano ${ }^{25}$. En esta provincia, Mélida conoció al pintor Eugenio Hermoso, quizás por mediación de Gestoso, ya que fue su alumno en la Escuela de Bellas Artes de Sevilla. Al parecer, Hermoso le estuvo mostrando lugares y piezas artísticas de interés a José Ramón Mélida, cuando visitó Fregenal de la Sierra en mitad de sus investigaciones para el catálogo; concretamente, le mostró dos curiosas pilas de agua bendita, que originariamente eran capiteles, uno corintio y otro compuesto, actualmente en la iglesia de Santa Catalina; un «arco árabe», que debe identificarse con la puerta de la casa que aparece en la lámina LXxiv del catálogo; y, por supuesto, el magnífico castillo de la localidad. Pero lo que más destacó Hermoso, y que le había gustado a Mélida «en extremo», fue una Virgen con el Niño (fig. 2), que el arqueólogo identificó como de «carácter entre francés y belga». Hermoso prometió mandarle un dibujo de la Virgen y fotografías a Gestoso ${ }^{26}$. En efecto, la promesa se cumpliría, y el 9 de octubre de este mismo mes recibió de parte de su antiguo alumno un dibujo de la escultura, de la cual proporcionaba datos básicos, como sus medidas $(129,5 \mathrm{~cm})$, que procedía del altar mayor del antiguo convento

25 BCC, FG, Correspondencia 1907-1908, n. ${ }^{\circ}$ 34, carta de José Ramón Mélida a José Gestoso, fechada en Madrid el 6/5/1907.

${ }^{26}$ BCC, FG, Correspondencia, 1909, vol. 2, n. ${ }^{\circ}$ 98, carta de Eugenio Hermoso a José Gestoso, fechada en Fregenal de la Sierra el 3/10/1909. 
de Santa Clara, del cual era patrona, y que tras la exclaustración fue trasladada a la iglesia de San José27.

Las conclusiones fueron claras para Gestoso cuando recibió una fotografía de la Virgen de parte de José Ramón Mélida ${ }^{28}$ y así se lo hizo saber poco tiempo después, a lo que Mélida respondió que estaba de acuerdo con Gestoso, aseverando que la Virgen de Fregenal es de artista borgonón, y que le haría gran favor si le comunicaba los datos documentales sobre esa obra, si es que los había ${ }^{29}$. Pero parece que Gestoso no pudo hallar ninguna noticia documental que ampliara la información de la que ya se disponía, por lo que se contentó con atribuirla a Mercadante de Bretaña, o al menos así lo expresó Mélida en el catálogo: «Es obra artística muy notable y según D. José Gestoso original del escultor Lorenzo Mercadante de Bretaña» (Mélida 1907-1911).

Mélida, que en 1914 se ocuparía del catálogo de Cáceres, no sería la única persona a la que auxiliaría Gestoso en estas tareas. A finales de 1908 y con el fin de iniciar los preparativos para el catálogo de Huelva, Rodrigo Amador de los Ríos comunicó a Gestoso su próximo viaje a Sevilla. También le consultó si había piezas procedentes de Huelva en el Museo Arqueológico Provincial, o en la colección del canónigo Francisco Mateos Gago, que se integraba en el Museo Arqueológico Municipal ${ }^{30}$. Gestoso era buen conocedor de las colecciones de los museos sevillanos, pues había fundado el Museo Arqueológico Municipal y había tomado contacto con las piezas del Arqueológico Provincial en su juventud ${ }^{31}$.

A instancias de esta pregunta sobre piezas procedentes de Huelva en Sevilla, José Gestoso contribuyó a la elaboración de este catálogo, con el suministro de una fotografía y un calco de la inscripción de una pieza que formaba parte de su colección de antigüedades ${ }^{32}$. Se trata de un fragmento cerámico, según Amador de los Ríos del tránsito del siglo XII al xIII, que posiblemente formara parte del brocal de un pozo, y cuyo molde originario sería de origen almohade (fig. 3). La pieza había sido adquirida por Gestoso a un vendedor que le indicó que había sido encontrada

${ }_{27}$ BCC, FG, Correspondencia, 1909, vol. 2, n. ${ }^{\circ}$ 107, carta de Eugenio Hermoso a José Gestoso, fechada en Fregenal de la Sierra el 19/10/1909. Mélida indica en el catálogo que la escultura medía $133 \mathrm{~cm}$.

${ }^{28}$ BCC, FG, Correspondencia, 1909, vol. 2, n. ${ }^{\circ}$ 163, carta de José Ramón Mélida a José Gestoso, fechada en Madrid el 7/12/1909; dicha fotografía se conserva en BCC, FG, Apuntes, t. I.

${ }^{29}$ BCC, FG, Correspondencia, 1909, vol. 2, n. ${ }^{\circ}$ 167, carta de José Ramón Mélida a José Gestoso, fechada el 13/12/1909.

${ }^{30}$ Francisco Mateos Gago había sido un importante anticuario y coleccionista de la Sevilla decimonónica, y su colección había sido adquirida por el Ayuntamiento para formar parte de los fondos del Museo Arqueológico Municipal.

31 BCC, FG, Correspondencia, 1907-1908, n. ${ }^{\circ} 310$, carta de Rodrigo Amador de los Ríos a José Gestoso, fechada en Madrid el 16/12/1908.

32 BCC, FG, Correspondencia, 1909 vol. 1, n. ${ }^{\circ}$ 158, carta de Rodrigo Amador de los Ríos a José Gestoso, fechada en El Escorial el 12/5/1909. 


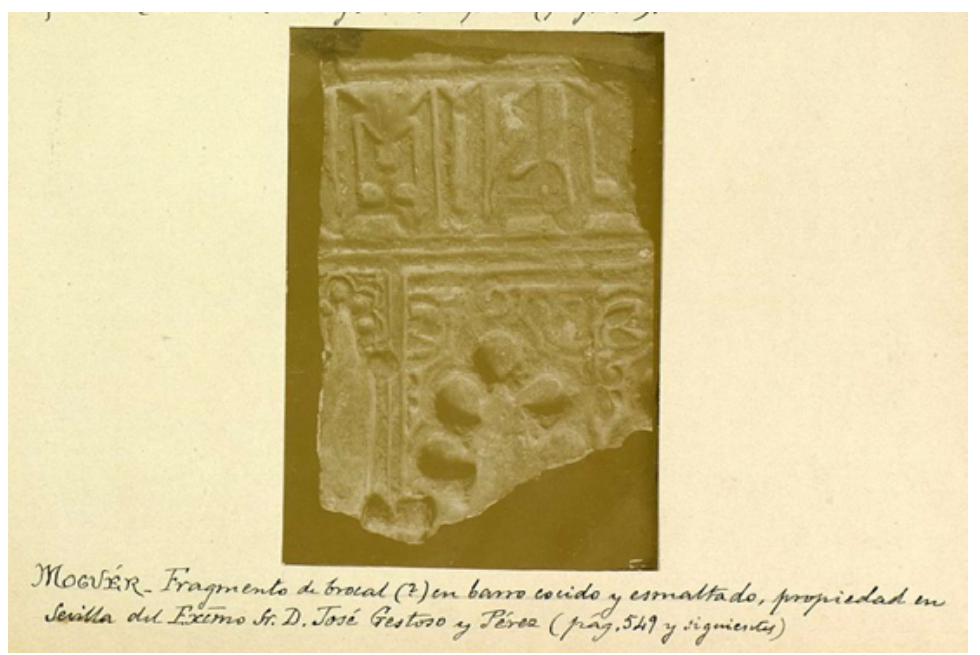

Fig. 3. Fotógrafo desconocido. Fragmento de brocal en barro cocido, Catálogo de los Monumentos Históricos y Artísticos de la provincia de Huelva, tomo de ilustraciones, s/n. @CCSIC, CCHS, Biblioteca Tomás Navarro Tomás.

en Moguer, de modo que este era el único testimonio con el que contaba Amador de los Ríos para incluirla en el catálogo monumental de Huelva ${ }^{33}$.

Enrique Romero de Torres, que además del catálogo de Cádiz se ocupó del de Jaén, también solicitó a Gestoso una lista con objetos procedentes de esta última provincia, que se encontraran en el Museo Arqueológico de Sevilla. De este autor, artista, como su hermano Julio, y conservador del Museo de Córdoba, del que llegó a ser director, parece que no guardaba una buena opinión Gómez-Moreno, que le llegó a llamar «aquel tipejo del Museo de Córdoba» (López-Yarto 2010). El investigador granadino demostró ser muy crítico con los autores de los catálogos; tampoco estaba convencido de la idoneidad de Rodrigo Amador de los Ríos. Cuando le adjudicaron la provincia de Málaga comentó: «Allá veremos lo que sale, pero me temo que sea deficiente en algunos casos, por ejemplo, en revisión de archivos, en lo romano, que allí tiene bastante interés, y en meterse por andurriales, pues ya está duro para trotes $\|^{34}$.

Ciertamente, Rodrigo Amador de los Ríos comprobó que en Málaga había mucho interés por el patrimonio de época romana; los estudios de Manuel Rodrí-

${ }^{33}$ La fotografía está incluida en el tomo correspondiente del Catálogo Monumental de Huelva, y fue merecedora de un minucioso análisis en la página 549 y siguientes.

${ }_{34}$ BCC, FG, Correspondencia 1907-1908, n. ${ }^{\circ}$ 17, carta de Manuel Gómez-Moreno a José Gestoso, fechada en Granada el 31/3/1907. 


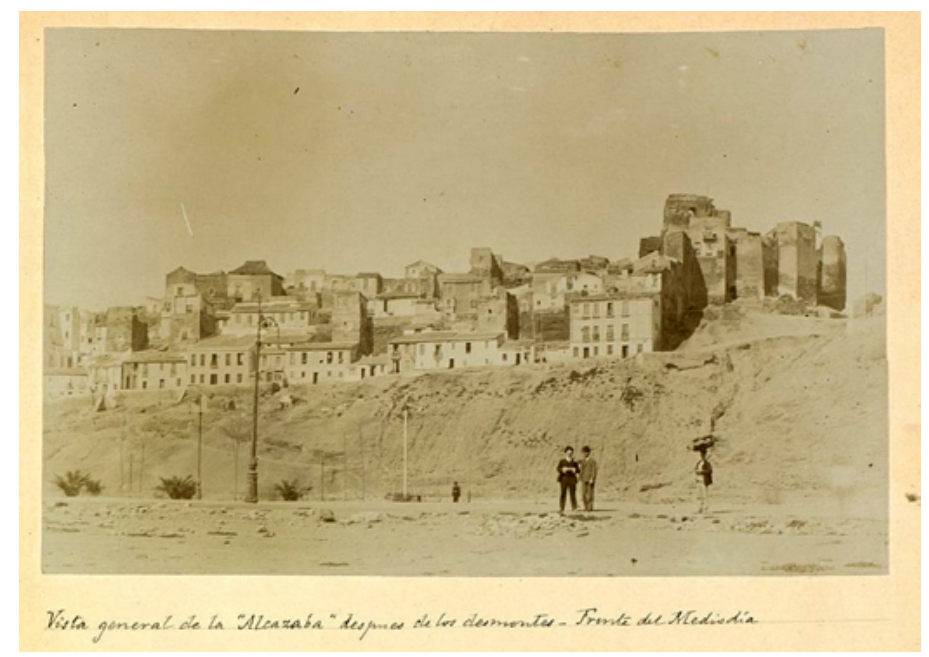

Fig. 4. Fotógrafo desconocido, alcazaba de Málaga después de los desmontes, ca. 1907. Catálogo de los Monumentos Históricos y Artísticos de la Provincia de Málaga, tomo 1, fotografías, fol. 14, (C)CSIC, CCHS, Biblioteca Tomás Navarro Tomás.

guez de Berlanga y el establecimiento del Museo Loringiano habrían contribuido a esa apreciación. En cambio seńalaba que eran pocos los restos existentes de época visigoda y también los de época "mahometana». Por sus cartas se percibe que no estaba entusiasmado con la ciudad, en la que decía que perdía el tiempo, y se mostraba muy crítico con la conservación de la alcazaba:

El convencimiento que los ruinosos torreones, algunos, son de origen romano en la Alcazaba, hacen el estudio de esta interesante, pero para V. que valía más que fuera demolido lo que existe en dicha alcazaba, la cual, por el estado en que se halla, es un borrón para Málaga. Por lo demás, poin-de-intèrêt de los malagueños ${ }^{35}$.

Es cierto que algunos años antes de que Amador de los Ríos estuviera en Málaga (1907) esta construcción había sido sometida a un proceso de desmonte en su zona baja (fig. 4), pero nada le podría haber hecho pensar que sería nombrada Monumento Nacional en 1931, ni que acabaría adquiriendo la imagen que luce en la actualidad, después de restaurarse en diversas campañas desde los años 30 del siglo $\mathrm{xx}$.

35 BCC, FG, Correspondencia 1907-1908, n. ${ }^{\circ} 23$, carta de Rodrigo Amador de los Ríos a José Gestoso, fechada en Málaga el 12/4/1907. 
Igual de sorprendente habría sido para Manuel Gómez-Moreno conocer la impresión que, según su autor, causó el catálogo de Málaga: «He tenido la satisfacción de que lo mismo la comisión que cuantos han visto mi trabajo, digan y hayan dicho, hasta los empleados del negociado de Bellas Artes del Ministerio, que no se ha presentado otro semejante, y que no esperan se presente ${ }^{36}$. Lo cierto es que se le concedió el catálogo de Huelva como recompensa a los servicios prestados en Málaga «ante lo cumplidamente que desempeñó su cometido» (López-Yarto 2010). Posteriormente se hizo cargo de la provincia de Albacete (1911), encomienda que tuvo que aceptar, a pesar de sus achaques ${ }^{37}$, pero de este, así como del de Barcelona (1913), no se han encontrado datos en el epistolario de Gestoso. También fue nombrado para ocuparse de Santander, pero este trabajo no lo llegó a empezar.

Después de haber comentado las noticias halladas sobre los catálogos monumentales de España en el epistolario de José Gestoso, es necesario hacer una aclaración respecto a la autoría del de la provincia de Sevilla. Ya hemos comentado que esta fue adjudicada en 1907 al arquitecto Adolfo Fernández Casanova, pero según los indicios que presentamos a continuación, parece que la propuesta se hizo en primer lugar a Gestoso (Casquete de Prado, 2016). Hay que recordar que la labor historiográfica de este personaje estuvo, prácticamente desde sus comienzos, marcada por la publicación de Sevilla Monumental y Artística (1899-1892), su obra magna, en la que, a lo largo de tres tomos, presenta un estudio sobre el patrimonio histórico-artístico de la ciudad de Sevilla y sus alrededores, desde la prehistoria hasta comienzos del siglo xix. Todas las investigaciones que se han dedicado al estudio del arte sevillano han bebido, de una forma o de otra, de esta fuente. Es tal el caudal de información que ofrece, ya sea de índole histórica, artística, o documental, que ha sido el máximo referente de la historiografía artística sevillana hasta tiempos recientes. Por tanto, las razones por las que Gestoso debía haberse ocupado de hacer el catálogo resultan más que evidentes. Además, fue un personaje bien relacionado, perfectamente integrado dentro de la cultura restauracionista, con contactos en Madrid, tanto políticos como académicos. Sin embargo, y a pesar de que todos los factores apuntaban hacia él como encargado para elaborar el catálogo de la provincia de Sevilla, no se contaba con su falta de disposición.

Los primeros tanteos que se produjeron para que aceptara este proyecto vinieron tempranamente de Rafael Ramírez de Arellano, cuando el proyecto de catalogación del patrimonio español se abrió a la participación de otros autores, aparte de Gómez-Moreno. En 1901 felicitó a Gestoso porque se había enterado de que le iban a encargar la formación de la «estadística monumental de esa provincia [Sevilla] $\aleph^{38}$. No consta documentación oficial sobre esta cuestión, aunque Gómez-

36 BCC, FG, Correspondencia, 1907-1908, n. ${ }^{\circ} 295$, carta de Rodrigo Amador de los Ríos a José Gestoso, fechada en Madrid el 30/11/1908.

${ }^{37}$ BCC, FG, Correspondencia, 1910, vol. 2, n. ${ }^{\circ}$ 92, carta de Rodrigo Amador de los Ríos a José Gestoso, fechada en Madrid el 16/12/1908.

${ }^{38}$ BCC, FG, Correspondencia, 1901-1902, n. ${ }^{\circ}$ 140, carta de Rafael Ramírez de Arellano a José Gestoso, fechada en Córdoba el 1/9/1901. 
Moreno era conocedor de la misma, pues aludió a la dificultad que supondría para cualquier persona este cometido: «Pensaba animarle para que no dejase el proyecto de catalogar su provincia, pero habiendo leido que hay en la diócesis mas de ¡mil! parroquias, ya es cosa de amilanarse, y no digo V., pero aun yo que tan andariego soy, me acobardaría de vérmelas todas por delante» ${ }^{39}$.

El tema no se volvió a mencionar en la correspondencia hasta más de un año y medio después. Mientras tanto, como ya hemos indicado, en 1902 Ramírez de Arellano fue propuesto por real orden para elaborar el catálogo de Córdoba. Un ańo después este investigador pudo saber, bien por Gestoso, bien por otros personajes relacionados con los catálogos, que su colega sevillano no se encontraba con fuerzas ni con salud como para llevar a cabo un trabajo de tal envergadura. Ciertamente, Gestoso sufría problemas digestivos (dispepsia) desde que era joven, y estos le provocaban temporadas de malestar, que generalmente le impedían la práctica de sus tareas cotidianas, y con más razón le supondrían un impedimento si la labor tenía que ser efectuada en una localización distinta a donde estaba su hogar. La enfermedad de Gestoso conmovió a Ramírez de Arellano, por lo que le propuso la elaboración conjunta del catálogo:

Me han hablado para hacerlo [el catálogo] en vista de la rotunda negativa de V. y he dicho que usted podría hacer la capital y yo la provincia. / Para la capital no tiene V. que viajar ni casi moverse de su casa. Cualquiera otro que la haga tendrá que valerse de su Sevilla Monumental y es lástima que venga otro, sea yo o cualquiera, a hacer una obra que va a tener mucha importancia sin más trabajo que espigar en el campo sembrado por V. Yo no lo consentiría tratándose de Córdoba y V. no debe consentirlo en Sevilla ${ }^{40}$.

Este aspecto es clave en su consideración como la persona idónea para este trabajo, pues no podía entenderse la catalogación del patrimonio histórico-artístico de la provincia sin consultar la Sevilla Monumental y Artística. Por ello, todo apunta a que Gestoso aceptó la oferta, ya que en una carta enviada por su amigo el político Guillermo de Osma se aprecian las gestiones que este practicó en Madrid para conseguir que se aceptara la doble autoría del catálogo junto a Ramírez de Arellano. Parecer ser que fue precisamente Osma quien originariamente transmitió al ministro de Instrucción Pública y Bellas Artes la negativa de Gestoso a elaborar el catálogo monumental de la provincia de Sevilla: «Cuando volví de mi última excursión a Andalucía tuve ocasión de decirle al Ministro que V. (que a todas luces era el indicado para hacer el Catálogo de Sevilla y tenia, por decirlo asi, hecho de antemano lo de su capital) en absoluto no quería encargarse». Ante la negativa de Gestoso, Osma

39 BCC, FG, Correspondencia, 1901-1902, n. ${ }^{\circ}$ 181, carta de Manuel Gómez-Moreno a José Gestoso, fechada en Granada el 7/1/1902.

40 BCC, FG, Correspondencia, 1903-1904, n. ${ }^{\circ} 46$, carta de Rafael Ramírez de Arellano a José Gestoso, fechada en Córdoba el 2/3/1903. 
concluyó que era posible que se pensara en Ramírez de Arellano, por su cercanía a la provincia y experiencia con el catálogo de Córdoba:

Es fácil que de resultas haya pensado el Ministro en darle ese encargo á Ramirez de Arellano, y deduzco de su carta de V. que así lo ha hecho ó lo piensa hacer. Me parece muy bien lo que Vds. han pensado. No sé si se ha hecho en alguna otra provincia pero no creo que pueda haber inconveniente. / Recuerdo que respecto de la de Toledo se habló de hacer alguna otra división del trabajo encargándose a dos personas, pero no sé si así se llegó a hacer.

Por si acaso, Osma se mostró cauteloso con este asunto, y prometió a Gestoso enterarse de lo que ocurriera al respecto, advirtiendo que, en todo caso, tuviera en cuenta que los nombramientos eran por real orden y a propuesta oficial ${ }^{41}$. Mientras tanto, Ramírez de Arellano animó a Gestoso a proponer el proyecto en instancias superiores, de la misma forma que él también tenía pensado hacerlo ${ }^{42}$.

Estos dos estudiosos comenzaron a planificar cómo llevar a cabo el catálogo, qué esquema seguir y, en suma, cómo organizar el trabajo. Ramírez de Arellano propuso el ya citado modelo de Gómez-Moreno y Riaño, que destaca por su claridad y estructura: la división entre arquitectura, escultura, pintura y artes industriales; enumerando cada uno de los objetos, y las partes diferentes de un edificio; y dentro de cada edificio, primero su historia y luego su descripción, también desglosando cada uno de sus ámbitos; por último, destacaba la presencia de «láminas, cortes verticales, planos de plantas, dibujos de perfiles, en fin, cuando dibujos y pormenores sean necesarios» ${ }^{43}$.

A pesar del interés de ambos por emprender el proyecto, nunca tuvieron la ocasión de materializarlo. Desconocemos las razones que motivaron la negativa del ministerio o de la Comisión Mixta de Monumentos, encargada de las propuestas, y no contamos con fuentes epistolares que aclaren estas circunstancias. Hasta 1907 no reaparece el tema del catálogo de Sevilla, en este caso, de la mano del ya citado Adolfo Fernández Casanova, quien se puso en contacto con Gestoso en dicho año para comunicarle su deseo de hacerse cargo del proyecto:

... anoche supe por Mélida que se está agotando el presupuesto destinado a formar el Catálogo monumental y artístico de cada provincia. / [...] No se me hubiera siquiera pasado por la imaginación el solicitar el nombramiento de la provincia de Sevilla, por reunir $V$. conocimientos muchos más generales que los míos en la materia, sino supiera que V. no quiere recorrer los pueblos de la provincia de Sevilla, y por eso y por la prisa que corre voy a escribir hoy mismo, o mañana, a más tardar, a Saa-

${ }^{41}$ BCC, FG, Correspondencia, 1903-1904, n. ${ }^{\circ}$ 55, carta de Guillermo de Osma a José Gestoso, fechada en Madrid el 15/3/1903.

${ }^{42}$ BCC, FG, Correspondencia, 1903-1904, n. ${ }^{\circ}$ 95, carta de Rafael Ramírez de Arellano a José Gestoso, fechada en Córdoba el 8/5/1903.

${ }^{43}$ BCC, FG, Correspondencia, 1903-1904, n. ${ }^{\circ}$ 95, carta de Rafael Ramírez de Arellano a José Gestoso, fechada en Córdoba el 8/5/1903. 
vedra, Catalina y Garrido que formase la Comisión Permanente (pues Fernández Duro está enfermo) para ver si quieren reservarme la provincia de Sevilla, si es que llego a tiempo. / Mas a pesar de eso, si V. pensase hoy de otra manera dígamelo con toda franqueza; pues caso de que los Sres. citados me contestasen favorablemente los volvería a escribir muy gustoso diciéndoles que le cedía a $\mathrm{V}$. el puesto por reconocer sinceramente que lo podía $\mathrm{V}$. desempeñar mucho mejor que yo ${ }^{44}$.

Ya hemos señalado que este arquitecto era muy amigo de José Gestoso, y al afecto que sentía hacia él habría que añadir el respeto profesional que le tenía. Es por ello por lo que se sintió con el deber moral de ofrecerle la tarea de catalogación, aun sabiendo que por razones de salud no la iba a aceptar. Tan pronto como obtuvo su nombramiento se lo comunicó a Gestoso, con el fin de viajar a Sevilla cuanto antes y "consultar con V. el plan mejor de presentación a las autoridades y de trabajo objeto de mi misión» ${ }^{45}$. Ya hemos visto la colaboración del erudito sevillano en otros catálogos, y en este caso, para Fernández Casanova, fue especialmente servicial suministrándole fotografías ${ }^{46}$. Como no podría ser de otra forma, las referencias a Sevilla Monumental y Artística son numerosas, aunque también a otras de sus obras como la Historia de los barros vidriados sevillanos (1903): «He terminado de redactar la sección de cerámica teniendo a la vista su excelente obra y he creído deber hacer constar lo que $\mathrm{V}$. y otros sevillanos han hecho para conseguir el nuevo renacimiento de tan bello arte» ${ }^{47}$.

En conclusión, las noticias que nos suministra el epistolario de José Gestoso sobre la elaboración de los catálogos monumentales de España nos llevan a confirmar las ideas que ya se habían expuesto acerca de este proyecto. En primer lugar, la ya conocida falta de medios materiales y personales para su ejecución, pero a la vez, la consideración de esta tarea para sus encargados como un honor, por encima del pesar del trabajo, y como una oportunidad para descubrir los tesoros artísticos del país. Por otro lado queda de manifiesto la pugna existente entre sus autores y los intentos de desacreditación entre ellos. También hemos podido apreciar las dificultades que este tipo de proyectos acarreaban para una sola persona. Y por último, hemos dado a conocer la intervención de José Gestoso en esta, aunque fallida, magna empresa cultural, en la que podría haber actuado con la responsabilidad intelectual que por su formación y conocimientos le habría correspondido.

Enviado: 31 de marzo de 2021; ACEPTAdo: 30 de abril de 2021

${ }_{44}$ BCC, FG, Cartas dirigidas al Excmo. Sr. Gestoso..., n. ${ }^{\circ}$ 161, carta de Adolfo Fernández Casanova a José Gestoso, fechada en Madrid el 16/4/1907.

45 BCC, FG, Cartas dirigidas al Excmo. Sr. Gestoso..., n. ${ }^{\circ}$ 161, carta de Adolfo Fernández Casanova a José Gestoso, fechada en Madrid el 29/6/1907.

46 BCC, FG, Cartas dirigidas al Excmo. Sr. Gestoso..., n. ${ }^{\circ}$ 182, carta de Adolfo Fernández Casanova a José Gestoso, fechada en Madrid el 16/1/1909.

47 BCC, FG, Cartas dirigidas al Excmo. Sr. Gestoso..., n. ${ }^{\circ}$ 182, carta de Adolfo Fernández Casanova a José Gestoso, fechada en Madrid el 14/10/1909. 


\section{BIBLIOGRAFÍA}

Argerich Fernández, I. (2012). «La fotografía en el Catálogo Monumental de España: procedimientos y autores», en López-Yarzo Elizalde, A. (coord.), El catálogo monumental de España (1900-1961): investigación, restauración y difusión. Madrid: Ministerio de Educación, Cultura y Deporte, pp. 107-124.

Borrás Gualís, G.M. (2006). «A modo de introducción: 100 años de Historia del Arte en España», en Borrás Gualís, G.M. y Pacios Lozano, A.R. (coaut.), Diccionario de historiadores españoles del arte. Madrid: Cátedra, pp. 13-34.

De Tena Ramírez, C. (2018). «El epistolario de José Gestoso (1852-1917) como fuente para el estudio de la historiografía artística española: índice de autores». Ars Longa. Cuadernos de Arte, Valencia: Universidad de Valencia, n. ${ }^{\circ} 27$, pp. 173-181.

Gómez-Moreno, M.E. (1983). «Prólogo», en Gómez-Moreno, M. Catálogo Monumental de la Provincia de Ávila. Ávila: Institución Gran Duque de Alba y Dirección General de Bellas Artes.

Gómez-Moreno, M.E. (1995). Manuel Gómez-Moreno Martínez. Madrid: Centro de Estudios Ramón Areces.

Hidalgo Brinquis, M.C. (2012). «Interpretación material de los Catálogos Monumentales de España», en López-Yarto Elizalde, A. (coord.), El catálogo monumental de España (19001961): investigación, restauración y difusión. Madrid: Ministerio de Educación, Cultura y Deporte, pp. 77-107.

López-Ocón Cabrera, L. (2012). «El papel de Juan Facundo Riaño como inductor del proyecto cultural del Catálogo Monumental de España», en López-Yarto Elizalde, A. (coord.), El catálogo monumental de España (1900-1961): investigación, restauración y difusión. Madrid: Ministerio de Educación, Cultura y Deporte, pp. 51-73.

López-Yarto Elizalde, A. (2010). El catálogo monumental de España (1900-1961). Madrid: Consejo Superior de Investigaciones Científicas.

López-Yarto Elizalde, A. (2012) «Los autores del Catálogo Monumental de España», en LópezYarto Elizalde, A. (coord.), El catálogo monumental de España (1900-1961): investigación, restauración y difusión. Madrid: Ministerio de Educación, Cultura y Deporte, pp. 37-48.

Lorenzo Arribas, J. y Pérez Martín, S. (2017). Excursiones zamoranas, 1903-1904. Epistolario de Manuel Gómez-Moreno y Elena Rodríguez Bolivar. Zamora: Editorial Semuret.

Mederos Martín, A. (2015). «Rodrigo Amador de los Ríos, trayectoria profesional y dirección del Museo Arqueológico Nacional (1911-16)». Spal, Sevilla: Editorial Universidad de Sevilla, n. ${ }^{\circ} 24$, pp. 183-209.

Mederos Martín, A. (2018) «La formación arqueológica y en historia del arte del joven Manuel Gómez-Moreno Martínez (1870-1899)», en España, S., Arranz, R. y Romero, A. (eds.) Colecciones, Arqueólogos, Instituciones y Yacimientos en la España de los siglos XVIII al XX. Oxford: Archaeopress, pp. 109-126.

Mélida Alinar, J.R. (1907-1911). Catálogo Monumental y Artístico de la Provincia de Badajoz. Original en http://biblioteca.cchs.csic.es/digitalizacion_tnt/index_interior_badajoz.html.

Morales, A.J. (1996). «El Catálogo de Bienes Muebles. Registro, conocimiento y tutela», en Catalogación del Patrimonio Histórico. Sevilla: Instituto Andaluz del Patrimonio Histórico, Junta de Andalucía, pp. 41-48. 
Muñoz Cosme, A. (2012). «Catálogos e inventarios del Patrimonio en España», en López-Yarto Elizalde, A. (coord.), El catálogo monumental de España (1900-1961): investigación, restauración y difusión. Madrid: Ministerio de Educación, Cultura y Deporte, pp. 13-36. 